\title{
LA SOCIEDAD Y LA ECONOMIA DEL CONOCIMIENTO EN LA GLOBALIZACIÓN*
}

\author{
Evangelina Zepeda García \\ da Universidad Marista de Querétaro
}

\begin{abstract}
Resumen: Se explica en qué consiste la Reforma del Estado y cómo se ve reflejada en los sistemas de educación superior de México y Brasil, en un contexto marcado por la sociedad y la economía del conocimiento.

Palabras clave: Reforma del Estado; Educación Superior; Política Educativa; Sociedad del Conocimiento
\end{abstract}

\section{INTRODUCCIÓN}

Dice lanni (1996) que cualquiera que estudie las ciudades en cualquier parte del mundo, debe analizar la situación, tomando en cuenta el entorno mundial en donde "el conocimiento acumulado sobre la sociedad nacional no es suficiente para esclarecer las configuraciones y los movimientos de una realidad que ya será siempre internacional, multinacional, trasnacional, mundial o propiamente global" (lanni, 1996, pág. 159).

En este contexto global, las políticas para la educación superior difícilmente son elaboradas a partir de un proyecto nacional, como se intentó en el siglo XX; las políticas actuales son producto de los movimientos económicos globales, porque las empresas del siglo XXI se instalan en cualquier parte del mundo siguiendo la conveniencia de sus negocios. Si las empresas no encuentran condiciones físicas, tecnológicas y/o humanas en un país, se mueven a otro(s) país(es) que sí las tenga. Los países, en consecuencia, se disponen a ofrecer a esas empresas mano y mente de obra para sus negocios. A mayor educación, mayor posibilidad de atraer a las empresas internacionalmente más competitivas, empresas que generarán empleo, productos, salarios, consumo. Los recursos naturales, las herramientas y la

\footnotetext{
* Artigo recibido en 05/11/2012 y aprobado en 05/12/2012.
} 
tecnología son secundarios porque lo más importante es el conocimiento, lo intangible en las personas que puede pasar a ser propiedad de las organizaciones con la propia aquiescencia de los sujetos porque para que la empresa pueda apropiarse de ese saber, necesita que el individuo esté en condición para generarlo y compartirlo.

En esta reconfiguración económica mundial, las personas educadas hacen ciencia, generan y usan tecnología y, si son administradas eficientemente, generan riqueza muy por encima de las materias primas o la mano de obra, "a diferencia del capital físico, el capital humano es potencialmente más maleable; los recursos humanos se pueden configurar una y otra vez para generar nuevas formas de conocimiento especializado" (Gibbons, 1994, pág. 27). Bien educado, un individuo puede modificar su manera de producir y administrar los recursos.

Peter Drucker señala, en Las nuevas realidades: en el Estado y en la política... en la economía y en los negocios... en la sociedad y en la imagen del mundo (1990), que en un mundo de grandes empresas, las más obvias limitaciones de la educación se hallan en la errónea premisa de preparar emprendedores o dirigentes sociales, porque en realidad la mayoría de los egresados trabajarán en organizaciones complejas. Si las escuelas se concentraran en enseñar las habilidades elementales para lidiar con el futuro laboral, la educación estaría sirviendo realmente a la sociedad.

El economista mexicano José Ayala Espino (2002) explica que una persona educada es más flexible, recupera información para cuestiones prácticas de manera ágil, al tiempo que utiliza materias primas y procesos no familiares de manera efectiva; "un bajo nivel educativo se convierte en una barrera que impide a los países aprovechar muchas de las oportunidades que brindan los mercados, debido a la carencia de mano de obra capacitada incrementando sus costos, desincentivando la inversión" (Ayala Espino, 2002, pág. 216).

Además, la idea se polariza; es insuficiente preparar al trabajador del siglo XXI; hay que prepararlo a lo largo de la vida, porque así como el conocimiento es obsolescente, el trabajador también lo es. El empleado es obsoleto una vez que aparece otro empleado más competente, el nuevo empleado sólo tendrá que demostrar que puede realizar lo que su antecesor y añadir valor a lo realizado, colocar su toque, innovar, plantear nuevas ideas, hasta que llegue otro que pueda hacer lo mismo que él y más. Dice Bauman (2007, pág. 39) que al empleado globalizado le corresponde adquirir las habilidades para comportarse de manera que "atraiga el favor de sus jefes y despierte en estos el interés por 'comprar' sus servicios y sus productos 
individualmente diseñados, del mismo modo en que los demás productores y vendedores seducen a sus posibles clientes para que deseen las mercancías que ponen a la venta" (Bauman, 2007, pág. 39).

A este escenario mundial la universidad pública brasileña llega fortalecida, con escasa participación de los distintos grupos sociales; la mexicana llega debilitada y con múltiples señalamientos. La fortaleza de la universidad brasileña es el resultado de veinte años de gobiernos militares, mismos que abrieron tempranamente el camino para la introducción del neoliberalismo, la preparación "ocurrió a través de la desarticulación de la esfera pública, de la restricción de derechos y de la despolitización" (Silva Franklin, 2006, pág. 199). En México, la politización vivida y protagonizada por la Universidad la colocó en la palestra de los políticos de nuevo cuño y en el descrédito de los grupos económicos, aunque, en los nuevos tiempos, la historia de las universidades esté perdiendo relevancia porque las políticas gubernamentales se aplican a todas ellas.

En la Lei de Diretrizes e Bases da Educação Nacional de 1968, aparecen varias de las políticas actuales de Brasil, tales como: la oportunidad de estudiar en las universidades públicas gratuitas más prestigiosas a los estudiantes académicamente más destacados; cobertura masiva en las instituciones privadas, de calidad y precios diversos, para atender a una población heterogénea; vinculación con el sector productivo; y otras relacionadas con las cuestiones laborales de los académicos y las prestaciones de servicios a los empresarios que decidan financiar la educación y la investigación. En México, poco a poco, se ha ido fortaleciendo la selección de los estudiantes académicamente más destacados para que se incorporen a las universidades públicas, aunque, a diferencia de Brasil, en México no es la clase económicamente más acomodada la que suele incorporarse a las universidades públicas.

\section{La Reforma del Estado y la política educativa}

Durante el siglo $\mathrm{XX}$, las crisis económicas hicieron necesaria la intervención del Estado para regular la economía; sin embargo, en la globalización, las empresas privadas son las protagonistas, no el Estado. Se presupone que la globalización "atenderá todos los rincones del planeta y cuidará, mediante políticas sociales, del bienestar de todos" (Sguissardi \& Dos reis Silva Júnior, 2009, pág. 27).

Con el nombre Reforma del Estado se conocen los ajustes que modifican la actividad estatal y que trata de confiar a la empresa privada las actividades realizadas por el Estado y, en el caso de las instituciones que 
continúan en manos del Estado, incorporar estrategias de empresa privada, considerando que la empresa privada sabe hacer negocios y sabe cómo alcanzar altos niveles de competitividad, a la altura de las circunstancias globales.

Décadas atrás, el Banco Mundial y otras corporaciones mundiales venían diseñando condiciones para alejar al Estado de la economía. En el Informe de la Comisión Trilateral elaborado por Michel Crozier, Samuel Huntington y Joji Watanuki, publicado originalmente con el nombre The Crisis of Democracy. Report on the Governability of Democracies to the Trilateral Comision, se destacaba que las condiciones de inestabilidad social e ideológica de los sesenta eran resultado del agotamiento del estado benefactor. El Estado se había vuelto incapaz, según el informe, de atender las demandas de una ciudadanía cada vez más participativa e interesada en los asuntos públicos. Los analistas identificaron una situación en la que a mayor participación ciudadana, mayor desconfianza hacia las instituciones; "para ellos, en consecuencia, era necesario restablecer el equilibrio entre el ejercicio del poder y la distribución de responsabilidades" (Acosta Silva, 2006), a fin de garantizar la vida democrática. Al tiempo que la participación ciudadana se tomó como estandarte para la reforma del Estado en los países desarrollados, en los subdesarrollados el problema económico y político se atribuyó al tamaño del Estado, a la discrecionalidad para aplicar los recursos y a la incapacidad para atender las demandas sociales, a pesar de haber contraído una enorme deuda pública.

John Williamson, investigador del Institute for International Economics, integró un decálogo sobre las políticas de libre mercado más recomendadas por las instituciones financieras internacionales en un documento que tituló "What Washington Means by Policy Reform", popularizado con el nombre de Consenso de Washington. En ese texto, Williamson abordó los problemas económicos en que estaba sumida América Latina, así como las soluciones que podrían ayudarle a salir de ella. Los grupos económicos de Washington asumieron que la crisis provenía de la aplicación de políticas de industrialización por sustitución de importaciones (mercado cerrado), y que habrían de establecerse políticas de corto plazo, considerando una reforma estructural para el largo plazo.

El decálogo consideraba tres soluciones generales, cada una con aspectos específicos: 1) restauración y estabilización fiscal, con reorientación del gasto público sólo para áreas estratégicas; 2) separación entre el Estado y la actividad económica; y 3) legalización de las privatizaciones. Según los organismos financieros, la causa de la crisis se hallaba en el crecimiento ex- 
cesivo del Estado, en el proteccionismo, en la regulación y en las abundantes e ineficientes empresas estatales. La solución estaba, consecuentemente, en un menor gasto del Estado con servicios públicos y en la separación entre el Estado y la economía, permitiendo que los particulares de cualquier parte del mundo invirtiesen donde mejor les pareciera.

Con la reorientación del gasto público se esperaba terminar con el populismo económico, alcanzar el equilibrio fiscal y la estabilidad macroeconómica (Williamson, 1990), basada en la eliminación de subsidios a los diferentes programas sociales, enfocándolos sólo hacia la educación y la salud. Esas eran las medidas generales para el corto plazo. Las reformas estructurales buscaban generar un modelo económico orientado al mercado y a la reducción de la participación del Estado. Si en años previos se habían cerrado las fronteras a mercancías extranjeras, ahora se indicaba abrirlas a la libre circulación. Se suponía que los productores nacionales podrían ofrecer sus productos en el exterior sin barreras arancelarias y traer dinero al país; de igual manera, la inversión extranjera llegaría a reactivar la economía, complementando la liquidez con la venta de las empresas estatales. Según los organismos asentados en Washington, una vez estabilizada la economía, por efecto de la liberalización y privatización de las empresas, se reactivaría el desarrollo y se pondría en marcha el crecimiento sostenido.

La Reforma del Estado en Brasil inició en los años 90, durante el gobierno de Fernando Collor de Mello, y se intensificó durante los cuatrienios de Fernando Enrique Cardoso con la transformación de la Secretaría de la Administración Federal en Ministerio de Administración Federal y de la Reforma del Estado (MARE), bajo la conducción de Luiz Carlos Bresser-Pereira, ex Ministro de Ciencia y Tecnología y ex Ministro de Hacienda, entre otros cargos ligados a la administración del Estado de São Paulo y de la Universidad de São Paulo. En México, la Reforma del Estado comenzó con Miguel de la Madrid y se intensificó durante el sexenio de Carlos Salinas de Gortari.

En Brasil, el fundamento legal para las privatizaciones se dio a través de a Carta Magna. En 1988, al promulgar la nueva Constitución, se abrió la posibilidad de incorporar al sector privado en el público. En 1995, vendría una modificación, específicamente en lo que se refiere a la participación de capital internacional en la provisión de servicios anteriormente públicos. Un movimiento más llegó en 1998 con la privatización y tercerización de la infraestructura que ofrecía servicio a las dependencias del Estado.

En México, al igual que en Brasil, se llevó a cabo el adelgazamiento estatal con cambios leves y paulatinos, pero constantes. En el sexenio de Miguel de la Madrid (1982-1988) se redujo el tamaño del sector público. 
Al principio del sexenio, había 1,216 empresas públicas, y al término había sólo 446; 722 fueron desincorporadas, de éstas, 260 fueron liquidadas, 136 desaparecieron, 80 se fusionaron, 28 se transfirieron y 218 se vendieron; y 48 se desincorporaron (Flores Alonso, 2003, pág. 91). Con Carlos Salinas de Gortari (1988-1994) continuó la privatización: hacia 1991, de las 446 empresas que quedaban, sólo había ya 239.

\section{TERCIARIZACIÓN Y DESCENTRALIZACIÓN}

En 1997, Bresser-Pereira, a cargo del Ministerio de Administración Federal y de la Reforma del Estado, subrayaba que el gobierno no quería desmontar al Estado, sino volverlo más eficiente (MARE, 1997, pág. 10); en su consideración, el Estado tiene que deshacerse de áreas no estratégicas para gobernar, como es el caso de los servicios no exclusivos del Estado: universidades, hospitales, museos, centros de investigación, los cuales deben continuar siendo públicos, aunque no administrados por el Estado, sino sujetos a observación del poder público a través de contratos de gestión, competencia y otros mecanismos de control social (Bresser-Pereira, 2001, pág. 16).

En la consideración de Bresser-Pereira, los servicios no exclusivos del Estado deben transformarse en organizaciones sociales que celebren contratos con el Poder Ejecutivo y cuenten con la autorización del legislativo para participar del presupuesto público (Bresser Pereira, 1996). Ya en la Constitución Política de 1988 se habían concebido "nuevos instrumentos de gestión y formas innovadoras de organización de la administración, procurando asimilar lo que hay más avanzado en las teorías y métodos de administración, en la experiencia internacional y en la propia trayectoria de administración pública brasileña" (MARE, 1997, pág. 7). Entre las medidas convertidas en ley se encontraban: la creación de Organizaciones Sociales sin vinculación administrativa con el Estado, pero operadas con los recursos del Estado; el fortalecimiento de la capacidad reguladora del Estado; la mejora en los servicios propios del Estado; la incorporación de mecanismos de evaluación; la readecuación de los cuadros de servidores públicos reajustándolos en número y perfil, y modificando los contratos de trabajo, particularmente las jubilaciones (MARE, 1997, págs. 8-9).

La propuesta de Brasil para reestructurar al Estado fue la delegación de actividades en grupos privados que operan con recursos públicos y privados, mediante la publicização, término empleado para referir "una operación mediante la cual lo público se privatiza a condición de que lo privado no sólo sea volcado a lo público, sino que se transforme a los criterios 
de lo público" (Reis Silva \& Sguissardi, 1999, pág. 10). Es privado porque lo proveen organizaciones privadas, y es público porque atiende necesidades públicas con recursos parcialmente públicos. El gobierno se arroga el derecho de evaluar el servicio proporcionado por las OSCIP y con ello la posibilidad de continuar o no entregándole recursos.

Funciona más o menos así: una institución ya existente, una universidad o un museo se transforma en organización social (OS); los trabajadores dejan de ser empleados del gobierno y se vuelven empleados de la OS; el Estado cede las instalaciones a la OS instituida como fundación de derecho privado. El Estado no se desatiende de ellas, siguen recibiendo recursos de la federación, del estado o del municipio en función de su productividad.

La Ley 9.790/99 dice que una OSCIP está conformada por personas jurídicas de derecho privado, sin fines de lucro: "se considera sin fines lucrativos la persona jurídica de derecho privado que no distribuye entre sus socios, asociados, consejeros, directores, empleados o donadores, eventuales excedentes operacionales, brutos o netos, dividendos, bonificaciones, participaciones o parcelas de su patrimonio obtenido mediante el ejercicio de sus actividades" (Lei № 9.790, 1999). Las OSCIP firman un contrato con el Poder Público en el que se establecen los derechos, responsabilidades y obligaciones de los firmantes. Las OSCIP deben rendir cuentas de los recursos asignados y atender evaluaciones periódicas en las que pueden perder su reconocimiento como OSCIP.

Además del financiamiento público, otras fundaciones pueden apoyar económicamente a las OSCIP. Una fundación está constituida por un patrimonio destinado a la realización de un fin, quien otorga el patrimonio establece tanto los objetivos como el funcionamiento de la fundación. De esta manera, las OSCIP reciben recursos del Estado con base en su desempeño, y pueden allegarse recursos a través de otros mecanismos tan variados como la cantidad de intereses relacionados.

En la cuestión educativa, por ejemplo, el gobierno de Brasil tiene como meta incrementar la cobertura de la educación superior y hacerlo con calidad. Con esa meta se han diseñado políticas que orientan las actividades de las instituciones educativas: si se ciñen a las disposiciones de gobierno, participan de los recursos económicos estatales; si además orientan sus actividades a los fines de alguna fundación, pueden recibir recursos de ella; $y$, si así conviene a sus intereses, pueden establecer un costo por el servicio que otorgan. En última instancia, podrían competir por recursos extraordinarios, atendiendo alguna política concreta. 
La Ley 10.973 dispone estímulos a la innovación y a la investigación científica y tecnológica, apoyando a organizaciones privadas y públicas para que realicen investigaciones en las Instituciones Científicas y Tecnológicas (ICT) públicas, y para que las personas que laboran en las ICT colaboren con esas empresas, recibiendo pago adicional. Se entiende que una ICT es un "órgano o entidad de la administración pública que tenga por misión institucional, entre otras, ejecutar actividades de investigación básica o aplicada de carácter científico o tecnológico" (Lei 10.973). En el artículo $4^{\circ}$ de la Ley, se apunta que las ICT podrán compartir sus laboratorios, equipos, instrumentos, materiales y demás instalaciones con micro y medianas empresas en actividades volcadas a la innovación tecnológica. También prevé la transferencia de recursos públicos para empresas y particulares que desarrollen innovación, aun cuando los productos sean destinados al lucro privado; se especifica que las empresas recibirán no sólo la condonación de impuestos, sino la explotación del o los productos y/o servicios desarrollados.

Mientras en Brasil predomina la conformación y consolidación del tercer sector (terciarización), en México se apuesta, por la privatización y la descentralización-centralización; esto es, privatizar todas aquellas empresas estatales que no ofrezcan obstáculos (o en su caso derribarlas económica o políticamente para privatizarlas), y lograr un mayor control estatal de aquellas dependencias de gobierno vía la evaluación y la rendición de cuentas.

La descentralización se refiere a delegar el poder con ciertas precauciones (Banco Mundial, 1997, pág. 12), de tal manera que se puedan aproximar los servicios a la sociedad, retirándolos del Gobierno Central y dejando la responsabilidad económica a estados y municipios, próximos a la sociedad, pues las determinaciones políticas siguen partiendo del centro.

\section{LA NUEVA GERENCIA PÚBLICA}

La privatización, la terciarización y la descentralización-centralización adelgazan al Estado, sin embargo, no todas las actividades de gobierno se pueden delegar; para las dependencias que continúan públicas se ha decidido la incorporación de técnicas gerenciales. La propuesta de la gerencia pública es el rediseño de la administración pública, introduciendo estrategias y valores de las empresas privadas: la planeación estratégica, la reingeniería de procesos, la gestión por resultados, la orientación al cliente, la evaluación y la certificación. En vez de asignar recursos a discreción, se supone la elaboración de planes y acciones verificables y medibles; en otras palabras, el diseño y operación de los procedimientos a partir de objetivos cuantificables y útiles según los requerimientos del entorno; una vez implementados los 
procesos, se revisa que no haya distorsión o desvío respecto a la meta. De haber cualquier alteración, se corrige para alinearlas de nuevo de acuerdo con los objetivos.

En Brasil, el Plan Nacional de Educación 2001-2010 estableció como objetivo incrementar la cobertura en la educación superior de un 12\% a un $30 \%$, aunque en esencia el problema podría no haber sido la cobertura, sino las estrategias para incorporar a un mayor número de estudiantes.

Según los datos presentados en el Plan Nacional de Educación 20012010, en 1998 había 973 instituciones (públicas y privadas) que ofrecían 776,031 cupos, de los cuales quedaban sin ocupar 124,674; a pesar de ello, en 10 años, se crearon 49 campi y 10 universidades públicas distribuidas por todo el país, además, se incrementó en más de 30\% el presupuesto para la educación superior, y la matrícula sólo creció 5\%. Por las políticas diseñadas por el gobierno federal, se puede inferir que el escaso crecimiento es atribuible a las características de los ingresantes y a los estándares solicitados por las universidades, como veremos más adelante.

En México, en el 2007, el Programa Sectorial de Educación 2007-2012 estableció como meta un 30\% de cobertura de educación superior en jóvenes de 18 a 24 años, en el VI Informe de Gobierno del Presidente Felipe Calderón se indica que esta cifra ha quedado superada. Según los datos proporcionados en el informe, hay un 32.8\%, aunque en esta cifra no se hace la distinción entre estudiantes del rango de edad para estar en ese nivel (19 a 23 años) y los que tienen menos o más edad.

En ambos países existe la preocupación por alcanzar mayor cobertura, aunque ante la dificultad de injerir directamente en las acciones de las universidades, los gobiernos establecen criterios para acceder a los recursos estatales; si las universidades quieren dinero, tienen que ceñirse a las reglas del Estado.

En diciembre de 2010, el Gobierno Federal de Brasil envió al Congreso el Plan Nacional de Educación 2011-2020 en el que se establece como meta "elevar la tasa bruta de matrícula en educación superior a un 50\%, y la tasa líquida para un 33\% de la población de 18 a 24 años, asegurando la calidad de la oferta", a partir de las siguientes estrategias:

1. Optimizar la infraestructura física y de recursos humanos en las instituciones públicas.

2. Ampliar la cobertura a un $33 \%$ en jóvenes de 18 a 24 años.

3. Elevar la tasa de conclusión a un $90 \%$, ofrecer un tercio de las vacantes en cursos nocturno y elevar a 18 los estudiantes por profesor. 
4. Ofrecer educación superior pública para la formación de profesores de educación básica.

5. Apoyar el ingreso de estudiantes de escuelas de educación media superior pública a las instituciones de educación superior públicas.

6. Incrementar el financiamiento a los estudiantes.

7. Asegurar al menos un $10 \%$ de los créditos curriculares en proyectos de extensión universitaria.

8. Fomentar la oferta y permanencia de los estudiantes.

9. Ampliar la participación de grupos vulnerables en la educación superior.

10. Garantizar legalmente el acceso.

11. Fomentar la articulación de actividades escolares con el mundo del trabajo.

12. Consolidar y ampliar la movilidad estudiantil y docente en lo nacional como en lo internacional.

13. Elevar la atención educativa de los grupos indígenas.

14. Mapear la demanda y fomentar la oferta de educación superior considerando las necesidades del país, la innovación tecnológica y la mejoría de la educación básica.

15. Incrementar el acervo digital.

16. Consolidar los procesos de selección.

Las 16 estrategias tienen en común un problema de origen: la selección de los estudiantes en las universidades públicas. Es sabido que el sistema universitario de Brasil tiene reconocimiento internacional por la alta producción científica de sus instituciones públicas; sin embargo a ellas acude una pequeña élite formada, consolidada y perpetuada en las propias universidades, situación que impide el ingreso de generaciones con características socioeconómicas diferentes. Las universidades públicas mantienen sus índices de calidad, seleccionando a los estudiantes más competentes y en número reducido -9 estudiantes por profesor (ANDES, 2007, pág. 24)- bajo la creencia de que a un mayor número de alumnos por profesor, menor calidad. Las universidades mexicanas admiten a un número más amplio de estudiantes; la UNAM atiende 13.6 estudiantes en promedio, lo cual contribuye al señalamiento de una universidad masificada con escasa calidad.

El vestibular, examen de selección brasileño, es el mejor filtro para los estudiantes menos aptos; es un examen que muy pocos consiguen aprobar. El vestibular establece un puntaje mínimo, si los aspirantes no alcanzan los 
puntos, quedan excluidos aunque el cupo quede sin ocupar. En México se llenan todas las vacantes y aún queda fuera un importante número de aspirantes que alcanzan los puntajes solicitados, esto es debido a que no hay cupos suficientes. En la Facultad de Ciencias Políticas y Sociales de la Universidad Nacional Autónoma de México, para la carrera de Ciencias de la Comunicación, se ofertaron 79 cupos, presentaron examen 2,434 aspirantes y resultaron seleccionados 84 que obtuvieron un puntaje mínimo de 98 aciertos de un total de 120 (Resultados del concurso 2012, UNAM).

La población que ingresa a las universidades públicas en México es de clase media y baja; el ingreso familiar de un estudiante de licenciatura en la UNAM, en promedio es de 2 a 6 salarios mínimos (UNAM, 2011). Las clases altas suelen estar en instituciones privadas cuya calidad no siempre es mala, incluso algunas son mejores que determinadas públicas según el ranking web de las mejores universidades. Por ejemplo, el Instituto Tecnológico y de Estudios Superiores de Monterrey se encuentra en segundo lugar de las mejores universidades de México. En Brasil, la universidad privada más importante es la Pontificia Universidad Católica, que se coloca en el $7^{\circ}$ lugar, siendo ocupados los primeros sitios por universidades públicas (Webometrics, 2010).

Con el conocimiento de que el vestibular es un filtro difícil de derribar, se han hecho esfuerzos por aplicar otros exámenes. El Examen Nacional de Educación Media (ENEM) fue creado en 1998 con el objetivo de evaluar el desempeño de los egresados de la educación media. Se pretende que a través de él se haga la vinculación entre los niveles medio y superior, pues el examen está orientado a evaluar 5 ejes cognitivos solicitados en el vestibular. Los estudiantes que están terminando sus estudios de nivel medio superior se postulan para realizar el ENEM, de aprobarlo, pueden aspirar a que se les asigne beca de PROUNI (Programa Universidad para Todos). Si no es para el PROUNI, puede que se le tome en consideración en alguna universidad pública y se le sume a su puntaje del vestibular, aunque las universidades públicas siguen sin aceptar que el ENEM sustituya a su vestibular, pues en su consideración el ENEM no tiene los mismos grados de exigencia.

El Sistema de Selección Unificada (SiSU), creado en 2009, es otro esfuerzo por desbancar al vestibular. El SiSU es realizado por el Ministerio de Educación (MEC) con él se pretende llenar las vacantes de las instituciones públicas participantes (universidades federales y estatales, tecnológicos e institutos). Los estudiantes se inscriben en el portal del SiSU, abierto por 5 días, eligen dos instituciones donde desean estudiar, una vez que cierra el sistema se asignan los lugares mediante proceso electrónico. Quienes tienen 
las notas más altas en el ENEM se quedan en la institución elegida como primera opción (si la institución seleccionada se adhirió voluntariamente al programa), hasta llenar el número de vacantes disponibles; luego se llenan las instituciones elegidas como segunda opción. Se publican los resultados y el estudiante tiene que acudir a matricularse. Si el estudiante no se presenta, se hace una segunda llamada a las notas que siguen. Así hasta que las vacantes sean completadas. Si un estudiante ya se había matriculado en su segunda opción sigue compitiendo; si se desocupó alguna vacante en su primera opción puede inscribirse en su primera elección y quedar desvinculado automáticamente de la segunda para permitir que otros participantes sigan concursando. El problema con la distribución de vacantes mediante el SiSU es doble, por un lado las instituciones públicas más prestigiadas no participan en el programa, lo cual deriva en el segundo problema, los estudiantes no están decididos a participar, esto se muestra en el número de veces que se hacen los llamados para ocupar las vacantes del SiSU.

Las universidades públicas recientemente están abriendo la posibilidad de estudiar en los cursos nocturnos, sin embargo las carreras impartidas en los horarios vespertinos no son las mismas que se imparten por la mañana, que valga decir, son las más solicitadas por los aspirantes.

En México, la distribución de los estudiantes se realiza desde el bachiIlerato, en un sistema parecido al SiSU, al menos en la zona metropolitana de la Ciudad de México. En 1996, 9 instituciones de nivel medio superior firmaron un acuerdo para aplicar un solo examen mediante el Concurso de Ingreso a la Educación Media Superior (COMIPEMS) y distribuirse a los aspirantes. Los aspirantes llenan un formato y seleccionan cuantas opciones deseen. Cada institución participante establece los criterios de admisión: puntaje mínimo en el examen y promedio general del nivel precedente. Los bachilleratos de la UNAM (preparatoria y Colegio de Ciencias y Humanidades) suelen ser los más demandados, y también los que más altos estándares ponen. Los estudiantes con menos puntajes y con menor promedio en secundaria suelen ser asignados a bachilleratos técnicos, tecnológicos o bimodales.

Con el COMIPEMS se busca garantizar el ingreso del mayor número de jóvenes a la educación media, jóvenes que, de tener buen promedio y acabar en el tiempo reglamentado, tendrán acceso al nivel superior. Vale decir, hay en México un esfuerzo por preparar a los jóvenes que más adelante estarán en las universidades, a diferencia de Brasil, en donde el estudiante tiene o no las competencias. La negativa de las universidades brasileñas para modificar sus lineamientos y admitir a un mayor número de estudiantes ha hecho con que se generen programas de incentivos para que abran sus puertas. La medida 
ha venido acompañada de la disminución de presupuesto, el congelamiento de los salarios a los profesores, la no contratación de nuevos docentes para cubrir las vacantes liberadas por los jubilados y los que renuncian, entre otras.

El Programa de Apoyo a Planes de Reestructuración y expansión de las Universidades Federales (REUNI) fue instituido mediante el Decreto 6.096 en abril de 2007. Es un programa de adhesión voluntaria mediante el cual se contempla ampliar la matrícula y garantizar la permanencia de los estudiantes, al tiempo que se combate el "desacuerdo entre la rigidez de la formación profesional y las amplias y diversificadas competencias demandadas por el mundo de trabajo y, sobre todo, los nuevos desafíos de la sociedad del conocimiento" (REUNI, 2007, pág. 8).

El Programa recibió, de 2008 a 2011, 2 billones de reales. Para recibir los recursos, las universidades tenían que inscribirse al Programa. Fueron repartidos entre las suscritas 1.5 billones de reales, con el compromiso de que introducirían los cambios señalados por el REUNI. Los 500 millones de reales restantes ( 0.5 billones) se entregaron con relación a la matrícula; por cada nuevo estudiante se asignaban 5,000 reales a las universidades. Al primer año ya se había adherido al programa el 100\% de las universidades. Los indicadores de éxito del REUNI se reflejarán en las evaluaciones del Instituto Nacional de Estudios e Investigaciones Educacionales Anísio Teixeira (INEP) y de la CAPES (Coordinación de Perfeccionamiento del Personal de Nivel Superior); después de 5 años de iniciado el programa, las universidades deberán tener una tasa de conclusión del $90 \%$ de los estudiantes de graduación y la atención de un promedio de 18 alumnos por profesor en cursos presenciales (Decreto 6,096, 2007).

A sabiendas de que el ingreso no es el único obstáculo para tener jóvenes académicamente preparados, se creó el Plan Nacional de Asistencia Estudiantil (PNAES), el cual busca evitar que los jóvenes de escasos recursos abandonen las universidades públicas federales para comenzar a trabajar o trabajar mientras estudian, en demérito de la calidad de sus estudios. El PNAES ofrece apoyo a las instituciones federales para que proporcionen a sus estudiantes de bajos ingresos casa estudiantil, alimentación, transporte, servicios de salud, acceso a las Tecnologías de la Información y la Comunicación, cultura, deporte, servicio de guardería y apoyo pedagógico. EI PNAES es complemento del REUNI.

En México, el Programa Nacional de Becas (PRONABES) es el instrumento del Gobierno Federal para apoyar económicamente a los jóvenes que ya se encuentran estudiando en las instituciones públicas del país. El PRONABES está dirigido a jóvenes de escasos recursos quienes, por 
su condición económica, podrían verse obligados a abandonar sus estudios. La institución receptora es la encargada de gestionar el apoyo. El PRONABES es el equivalente al PNAES brasileño, sólo que en el PRONABES en lugar de entregar los recursos a las instituciones educativas receptoras, los entrega a los estudiantes de manera directa. El monto en el primer año de estudios es de 750 pesos mensuales ( 118 reales, aproximadamente), en el segundo de 830, el tercero de 920 y el cuarto y el quinto de 1000 pesos (la beca se suspende durante el período vacacional en algunas localidades). El PRONABES se otorga a cualquier estudiante que lo solicite, independientemente del promedio que haya obtenido en el nivel inmediato anterior; sin embargo para mantenerla es necesario que no repruebe ninguna asignatura y que a partir del 3er año tenga un promedio mínimo de 8.

Los beneficios del PRONABES, del REUNI y del PNAES se otorgan a estudiantes de Instituciones de Educación Superior guiadas por las políticas federales. En Brasil, las universidades públicas deciden si se incorporan o no al programa ofrecido, finalmente representan recursos extraordinarios. En México, las universidades deben ceñirse a determinadas evaluaciones para poder acceder a recursos federales extraordinarios, sea para sus profesores, sus estudiantes o las propias instituciones.

En el Programa Sectorial de Educación (SEP, 2007) se tiene contemplado que para el 2012 , un $72 \%$ de los profesores de tiempo completo de las universidades publicas mexicanas tomen cursos de actualización, y se llegue a un $70 \%$ de eficiencia terminal de estudiantes; además de incrementar el número de estudiantes matriculados en programas de calidad. Se consideran programas de calidad aquellos que alcanzan el nivel 1, nivel otorgado por los Comités Interinstitucionales para la Evaluación de la Educación Superior (CIEES).

Las políticas evaluadoras en México comenzaron con el Programa para la Modernización Educativa 1989-1994; sin embargo, hasta la fecha no existe un Sistema Nacional de Evaluación como el Sistema Nacional de Evaluación de la Educación Superior (SINAES) de Brasil. En México, hay varios programas, organismos y fondos orientados a la evaluación, es el caso del Fondo para la Modernización de la Educación Superior (FOMES), el Programa para el Mejoramiento del Personal Académico (PROMEP), los Comités Interinstitucionales para la Evaluación de la Educación Superior (CIEES), el Centro Nacional de Evaluación para la Educación Superior (CENEVAL), el Consejo para la Acreditación de la Educación Superior (COPAES), el Padrón Nacional de Posgrado SEP-CONACYT (PNP), el Sistema Nacional de Investigadores (SNI), el Sistema de Acreditación Institucional de la Federación 
de Instituciones Mexicanas Particulares de Educación Superior (FIMPES), entre otros.

Los Comités Interinstitucionales para la Evaluación de la Educación Superior (CIEES) realizan una evaluación por pares; se considera par a quien ha destacado en un área específica, y por tanto tiene las cualificaciones para realizar la evaluación. La institución que desea ser evaluada hace la solicitud y agenda una cita para ser visitada. Previo a la visita, la institución entrega un informe de autoevaluación en el que indica las normas bajo las cuales opera, la relación de estudiantes, los volúmenes en la biblioteca y el porcentaje de asignaturas para cada plan de estudios. Los evaluadores deciden si los salarios son adecuados, si la legislación es pertinente (léase parecida a otras ya aceptadas), número de profesores de tiempo completo, laboratorios, bibliotecas, etc. Si la institución no cumple con todos los requisitos solicitados, los evaluadores emiten recomendaciones para que en un determinado plazo sean modificados. Las instituciones evaluadas tienen la obligación de pagar los honorarios, viáticos y talleres que el CIEES les requiera.

No existe una periodicidad para evaluar los programas, se hace cuando alguna institución requiere la calificación que la vuelve merecedora del presupuesto federal o porque puede concursar por fondos. A estas evaluaciones las IES se adhieren de manera voluntaria porque así conviene a sus intereses.

Los CIEES son la antesala para la evaluación pública del Consejo para la Acreditación de la Educación Superior (COPAES), reconocido por la SEP para acreditar programas académicos. La cuestión es que "los criterios que establecen los CIEES para evaluar el programa de estudios de cualquier universidad no son los que utiliza el COPAES para acreditar el mismo programa" (Leal, 2008).

Si la institución quiere tener acceso a recursos extraordinarios como los del PROMEP (Programa de Mejoramiento del Profesorado) tiene que ceñirse a determinados programas. El PROMEP se creó en 1996 como resultado de un análisis Ilevado a cabo por la Secretaría de Educación Pública en la década de los noventa. En el análisis se encontró que en el Sistema Nacional de Educación superior había un alto porcentaje de profesores que no contaban con el nivel académico adecuado (doctorado), ni articulado en las tareas de investigación (Diario Oficial, 2009). Desde 1996, se otorga a los docentes recursos para continuar sus estudios de posgrado tanto en universidades nacionales como extranjeras, e incentivos económicos a quienes teniendo posgrado posean el perfil deseable que consiste en la generación y aplicación del conocimiento (investigador), participación 
en actividades de tutoría (tutor), ejercitación de la docencia (profesor) y participación en la gestión académica (gestor) (PROMEP, 2010).

Los estímulos para profesores con perfil deseable son asignados según las consideraciones de los propios profesores convertidos en gestores; los docentes evalúan a sus pares y deciden si son candidatos. No hay un organismo encargado de la evaluación, el candidato a los estímulos se convierte en juez y parte. Una de las quejas más frecuentes se refiere a la inexistencia de parámetros claros sobre los puntos evaluados y la importancia que cada uno de ellos tiene en la evaluación.

Además del PROMEP, el Sistema Nacional de Investigadores (SNI) evalúa a tecnólogos e investigadores para otorgarles reconocimiento y apoyo económico. La evaluación se realiza a través de pares, y el fin es dar título de investigador nacional en alguno de los niveles otorgados por el SNI. El SNI fue creado en 1984 para evaluar la producción de los investigadores y establecer estímulos económicos conforme a su desempeño. Es una evaluación que se realiza a cada 4 años para los integrantes del sistema, y"además de estímulos económicos, distribuye símbolos de prestigio" (Suárez Sosaya \& Muñoz García, 2004, pág. 134).

En Brasil, el mejor estímulo para el docente es la legislación, que le permite desarrollar, al mismo tiempo, los mismos proyectos para una universidad y para cualquier empresa, incluso el docente tiene la posibilidad de acceder a la explotación del bien trabajado, si de tecnología se trata. El Sistema Nacional de Evaluación de la Educación Superior (SINAES) se aplica a las universidades privadas con el objetivo de mantener en ellas un grado mínimo de calidad; si las instituciones aprueban, entonces pueden verse beneficiadas con la incorporación de alumnos becados por el Estado, quien habrá de pagar la colegiatura mediante la exención de impuestos, como lo marca el PROUNI. En la tabla 1 se observa que el $74 \%$ de la matrícula se encuentra en instituciones privadas.

El Programa Universidad para Todos (PROUNI), iniciado en 2004, está diseñado para otorgar becas a estudiantes que se incorporen a las instituciones privadas. Los aspirantes a estos recursos deberán demostrar ingresos familiares menores a tres salarios ( $\mathrm{R} \$ 570)$, además de haber cursado la enseñanza media en institución pública o privada con beca de $100 \%$ o ser docente de educación básica. La beca se entrega a partir de los resultados del ENEM (Examen Nacional de Educación Media) y el estudiante no requiere pasar por la evaluación de ingreso (vestibular) de la institución receptora. La beca consiste en recibir el descuento del 100 o del $50 \%$ del cobro de la matrícula, a cambio la institución receptora queda exenta de pagar impuestos. 
Tabla 1 - Evolución del número de matrículas de 2001 a 2010 (Censo da Educação Superior, 2011)

\begin{tabular}{|c|c|c|c|}
\hline Año & Privadas & Públicas & Total \\
\hline 2001 & $2,091,529$ & 944,588 & $3,036,113$ \\
\hline 2002 & $2,434,650$ & $1,085,977$ & $3,520,627$ \\
\hline 2003 & $2,760,759$ & $1,176,174$ & $3,936,933$ \\
\hline 2004 & $3,009,027$ & $1,214,317$ & $4,223,344$ \\
\hline 2005 & $3,321,094$ & $1,246,704$ & $4,567,798$ \\
\hline 2006 & $3,632,487$ & $1,251,365$ & $4,883,852$ \\
\hline 2007 & $3,632,487$ & $1,251,365$ & $5,250,147$ \\
\hline 2008 & $4,255,064$ & $1,552,953$ & 5.080 .017 \\
\hline 2009 & $4,430,157$ & $1,523,864$ & $5,954,021$ \\
\hline 2010 & 4.736 .001 & 1.643 .298 & 6.379 .299 \\
\hline
\end{tabular}

Las instituciones privadas participantes firman (por 10 años) convenio para cubrir un $10 \%$ de su matrícula con becados. No participan en el Programa las instituciones que obtienen calificación de insuficiente en el SINAES. Si la institución reprueba la evaluación por dos años consecutivos, debe mantener la beca de los estudiantes que hayan ingresado mientras acreditó la evaluación.

El Fondo de Financiamiento al Estudiante de Educación Superior (FIES) otorga recursos a estudiantes de escuelas particulares acreditadas por el SINAES. El estudiante hace la solicitud, elige el banco con el cual quiere contratar el crédito y la Caja Económica Federal valida la información del estudiante para solicitar el crédito. De ser aprobado, el banco entrega la colegiatura directamente a la institución educativa.

No todos los estudiantes tienen posibilidad de acceder al FIES. Primero, deben demostrar que tienen un porcentaje de sus ingresos'libre'. Por ejemplo, si la colegiatura mensual es de $\mathrm{R} \$ \mathbf{6 0 0 . 0 0}$, debe ganar al menos $\mathrm{R} \$$ 1,000 (poco más de dos salarios mínimos). Además, el solicitante está obligado a presentar a un fiador con una renta mensual similar, o bien presentar 3 a 5 fiadores (fiadores solidarios), todos ellos solicitantes de FIES, quienes se comprometen a pagar entre ellos si alguno falla. Los estudiantes beneficiarios parciales de PROUNI quedan exentos de presentar fiador.

El estudiante paga intereses desde el momento en que recibe el crédito: $\mathrm{R} \$ 50$ trimestralmente. Concluidos sus estudios, tiene 18 meses para comenzar a pagar su crédito, en tanto, sigue pagando los mismos intereses 
mensuales que mientras estudiaba ( $\mathrm{R} \$ 50$ trimestrales). Después de los 18 meses, el total del financiamiento se divide entre 13 años y se le agrega un año pagando la cantidad mensual, por los intereses. Al crédito recibido es necesario sumarle el $3.4 \%$ de interés anual.

A principios de 2012, en México, el presidente Felipe Calderón presentó el Programa Nacional de Financiamiento a la Educación Superior, con el que se pretende financiar a más de 23,000 jóvenes en todo el país, se prevé otorgar hasta 215,000 pesos por estudiante de nivel licenciatura; el único requisito es que el interesado haya sido aceptado en alguna universidad incorporada el programa. El crédito se brinda para pagar hasta en 15 años, aunque no es pública la tasa de interés.

\section{CONSIDERACIONES FINALES}

Brasil avanza, promueve la innovación, la competencia y la competitividad; establece normas, estrategias y acciones que orientan al Estado, a las universidades y a los individuos a incorporarse a la globalización; pero las oportunidades no son para todos, sólo son para los más aptos. Su sistema exclusivo y excluyente lo hace tener uno de los mejores sistemas de educación superior en América Latina y refleja fielmente lo dicho por los organismos internacionales en torno a la educación Superior. Es en Brasil donde el empleo para los egresados de educación terciaria incrementa en un $91 \%$ para los hombres y un $82 \%$ para las mujeres, además de que sus ingresos se incrementan en más del 100\%, según reporta la Nota para Brasil hecha por la OCDE; sin embargo para ingresar a una universidad pública, especialmente, se debe tener un excelente desempeño académico, y para ingresar a una escuela particular, hay que tener recursos económicos, de lo contrario no se pueden mantener en el sistema.

Históricamente la exclusión por género y raza está presente en la sociedad brasileña. Negros y mulatos forman el $45 \%$ de la población y ganan menos que los blancos. Las mujeres son mayoría en el país, pero no son bien aceptadas en el ámbito laboral. Los estados del sur, habitados en gran medida por inmigrantes europeos, son de los más ricos,mientras que los estados del norte, con mayoría de población indígena y negra, son de los estados más pobres. Brasil es reconocido por ser uno de los países más desiguales del mundo, en el que el $10 \%$ de la población tiene ingresos superiores en un $47 \%$ al del $10 \%$ de la población más pobre y poco más del $20 \%$ de su población vive en situación de indigencia. En 2010, el PIB se multiplicó en un 110\%, con un ingreso semejante a países como Japón, Finlandia y Noruega, según datos del Instituto Brasileño de Geografía y Estadística (IBGE, 2010), pero es 
el $4^{\circ}$ país más desigual en América Latina, y se le llega comparar con niveles de desigualdad de Namibia, Sierra Leona, Suazilandia, Botsuana y República Centroafricana, los cinco países con mayor desigualdad en el mundo.

Durante el período presidencial de Lula da Silva, la situación cambio ligeramente. En cuatrienios anteriores, la población en condiciones de pobreza estaba por encima del 50\%, con Lula da Silva el 19\% de esa población mejoró su situación económica gracias a programas asistencialistas. Actualmente $57 \%$ de los brasileños vive en una familia que recibe algún tipo de apoyo gubernamental, según datos del IBGE (2010). En contraste con la situación social de la amplia mayoría, "Brasil desarrolló en los últimos cuarenta años un sistema de posgraduación e investigación que es considerado, de manera general, como de muy buena calidad, confirmando la tradición elitista del país, al invertir fuertemente en áreas de punta, pero sin conseguir atender de forma satisfactoria y amplia a su población"(Schwartzman, 2005, pág. 15). Con todo y las medidas implementadas por Lula da Silva y ahora por Dilma Rousseff, el problema educativo queda muy lejos de ser resuelto por el problema de la pobreza; "una cuarta parte de los jóvenes brasileños entre 12 y 24 años son tan pobres que no podrían mantenerse en una institución de educación superior, aunque ésta fuera gratuita" (Dias Sobrinho \& Brito, 2008, pág. 494).

Habría que continuar observando la situación de Brasil pues la Reforma del Estado llevada a cabo dista de otras Reformas en la región. Tanto Fernando Enrique Cardoso como Luiz Inácio Lula da Silva optaron por las OSCIP (Organizaciones de la Sociedad Civil de Interés Público) para dar fuerza a las privatizaciones y hacer uso racional de los recursos del Estado sin dejar todo en manos del sector empresarial, sino en organizaciones sociales constituidas sin fines lucrativos y con rendición de cuentas dado que los recursos económicos son proporcionados por el Estado. Habría que seguir a Brasil también en la ampliación de la matrícula de educación a nuevos grupos sociales pues al incrementar la competencia podría verse afectada la posibilidad de conseguir un empleo, y más todavía conseguir un empleo que supere en más del 100\% el salario de trabajadores con menos años de estudio.

En México, no debemos olvidar que la deuda histórica con los trabajadores se encuentra en los salarios, y no interesa cuál sea su nivel de preparación académica. Una persona, aun teniendo todos los grados educativos, carece de certidumbre en sus ingresos y por ende al acceso a los bienes y servicios del sistema. La principal crítica para el Estado mexicano es que ha ido disminuyendo su participación en la economía del país, desligándose del impulso, promoción y cumplimiento de las necesidades educativas, y aunque, a través de políticas públicas, va impulsando cambios en las Instituciones de Educación Superior (IES), de manera general se 
desatienden y desconocen las necesidades del país. Como apuntan (Suárez Sosaya \& Muñoz García, 2004, pág. 25):

Los mexicanos vivimos en un escenario social y político cuyos espacios institucionales públicos están siendo derrumbados, para abrirle paso a la privatización y a la apertura económica. Sin embargo, la política estatal no ha dado muestra de estar atendiendo los aspectos esenciales de la protección que debe el Estado a los más débiles y al país en su conjunto, para que no sucumban frente al dominio de las corrientes globalizadoras.

El aspecto educativo, nodal en las transformaciones económicas mundiales, carece de rumbo congruente y está llevando a problemas difíciles de erradicar. La privatización y la ampliación de la cobertura de la educación superior se encaminan a la generación de empleados calificados que poco colaborarán en la generación de nuevos conocimientos en un sistema incapaz de generar empleo para los profesionales que año con año egresan de las instituciones de nivel superior. La masificación educativa de un país que no promueve ni asegura la inversión estatal o particular genera exceso de trabajadores calificados que sólo egresan para engrosar las filas de los desempleados.

La OCDE, en el Panorama de la Educación 2011, en la nota sobre México, señala que el desempleo en México no está relacionado con la educación. Tampoco hay diferencia, al menos para el desempeño educativo, entre una institución pública y una privada. Según la misma fuente, la situación es peor para las mujeres, quienes con grados académicos de maestría o doctorado tienen menos posibilidades de conseguir un empleo. Entre mayor nivel de estudios, la dificultad para conseguir empleo se intensifica. Las empresas particulares no alcanzan a dar cabida a los licenciados, maestros y doctores de todas las áreas, ni las universidades ni el gobierno abren la oportunidad. Cada año egresan en promedio 3.000 nuevos doctores en México, 50\% de ellos se encuentra desempleado y sólo 3 de cada 100 consigue un empleo fijo y bien remunerado en el sector público, según Arturo Menchaca Rocha, presidente de la Academia Mexicana de Ciencias (2011).

\section{SOCIETY AND THE ECONOMICS OF KNOWLEDGE IN GLOBALIZATION}

ABSTRACT: State reform is explained and how this is reflected in Higher Education systems in Mexico and Brazil, in a context characterized by society and the economics of knowledge.

KeYwords: State Reform. Higher Education. Educational Policy. Society and the Economics of Knowledge. 


\section{A SOCIEDADE E A ECONOMIA DO CONHECIMENTO NA GLOBALIZAÇÃO}

REsumo: Explica-se em que consiste a Reforma do Estado e como ela se vê refletida nos sistemas de educação superior do México e Brasil, em um contexto marcado pela sociedade e a economia do conhecimento.

Palavras-chave: Reforma do Estado. Educação Superior. Política Educativa. Sociedade do Conhecimento.

\section{REFERENCIAS}

ACOSTA SILVA, A. (2006). "Perspectivas del Debate a Veinte Años del Reporte a la Comisión Trilateral". Recuperado el 20 de noviembre de 2012, de Gobernabilidad y Democracia: http://www.robertexto.com/archivo17/gobernab_democrac.htm\#3

ANDES. (2007). As novas faces da reforma universitária do governo Lula e os impactos do PDE sobre a educação superior. Brasília, Distrito Federal: Cadernos Andes.

AYALA ESPINO, J. (2002). Fundamentos institucionales de mercado. Distrito Federal, México: UNAM, DGAPA, Facultad de Economía.

BANCO MUNDIAL. (1997). Informe sobre el Desarrollo Mundial, el Estado en un mundo en transformación. Washington, D.C.: Banco Mundial.

BAUMAN, Z. (2007). Los retos de la educación en la modernidad líquida. Barcelona, España: Gedisa.

BRESSER PEREIRA, L. C. (1996). Crise econômica e reforma do Estado no Brasil - para uma nova interpretação da América Latina. São Paulo: Editora 34.

BRESSER-PEREIRA, L. C. (2001). “Uma nova gestão para um novo Estado: liberal, social y republicano". Revista do Serviço Público (52), 5-24.

CENSUP, INEP, Ministério de Educação, Governo Federal do Brasil. (Outubro, 2011). "Censo da educação Superior 2010, divulgação dos principais resultados do Censo da Educação Superior 2010". Brasília.

DIAS SOBRINHO \& BRITO. (2008). "La educación Superior en Brasil: principales tendencias y desafíos". Revista da Avaliação da Educação Superior, 487-507.

DRUCKER, P. (1992). The age of discontinuity: Guidelines to our changing society. Transaction Publishing.

DRUCKER, P. (1997). Sociedade pos-capitalista. São Paulo: Pionera, Novos Umbrais.

FLORES ALONSO, M. (2003). "Reforma del Estado y reforma administrativa”. Recuperado el 2011 de noviembre de 03, de Centro de Estudios Sociales y de Opinión Pública: http:// www.diputados.gob.mx/cesop/doctos/reforma.pdf

GIBBONS, L. N. (1994). The new production of knowledge. London, England: Sage Publications. 
GIBBONS. (1998). "Pertinencia de la Educación Superior en el Siglo XXI”. Conferencia Mundial sobre la Educación Superior de la UNESCO.

IANNI, O. (1996). Teorías de la globalización. Distrito Federal, México: Siglo XXI, CEIICH-UNAM.

IBARRA COLADO, E. (2008). “Exigencias y desafíos de la organización y la gestión de la universidad: pasado político, mercados presentes, (im-) posibles futuros". Seminario Interinstitucional la Universidad en el México de Hoy. Distrito Federal, México: Instituto de Investigaciones Económicas, UNAM.

MARE. (1997). A Reforma do Aparelho do Estado e as Mudanças Constitucionais: Síntese \& Respostas a Dúvidas mais Comuns. Brasília: MARE.

Portal de estadística universitaria (2010), disponible en http://www.estadistica.unam. $\mathrm{mx} /$ indicadores/.

POY SOLANO, L. (20 de mayo de 2010). “Expertos: mediática, la estrategia de SEP sobre la meta de cobertura universitaria". La Jornada, pág. 41.

PRESIDÊNCIA DA REPÚBLICA, Casa Civil Subchefia para Assuntos Jurídicos. (1999). Lei $N^{\circ}$ 9.790. Brasília.

PRESIDÊNCIA DA REPÚBLICA. (1996). LDB - Lei de Diretrizes e Bases da Educação Nacional. Lei 9.394. Brasilia.

PRESIDÊNCIA DA REPÚBLICA. (2004). Lei de Incentivos à inovação eà pesquisa científica tecnológica (10.973). Brasília.

PROMEP. (2010). Secretaría de Educación Pública. Recuperado el 30 de octubre de 2010, de Subsecretaría de Educación Superior: http://promep.sep.gob.mx/

PRONABES. (2010). Secretaría de Educación Pública. Recuperado el 7 de noviembre de 2011, de http://pronabes.sep.gob.mx/index.html

REIS SILVA \& SGUISSARDI. (1999). Novas faces da educação superior no Brasil. Bragança Paulista: EDUSF.

REPÚBLICA FEDERATIVA DO BRASIL. (2007). Decreto 6,096

Resultados del concurso junio de 2012, UNAM. Disponible en https://servicios.dgae. unam.mx/Junio2012/convocatoria.html.

REUNI. (2007). Reestruturação e expansão das universidades federais. Diretrizes gerais. Brasília: Presidência da República.

SCHWARTZMAN, S. (2005). Os desafios da educação no Brasil. En Brock \& Schwartzman. Rio de Janeiro: Nova Frontera.

SECRETARÍA DE EDUCACIÓN PÚBLICA. (2007). Programa Sectorial de Educación 20072009. Distrito Federal, México: SEP.

SGUISSARDI \& DOS REIS SILVA JÚNIOR. (2009). Trabalho intensificado nas federais. Pós-graduação e produtivíssimo académico. São Paulo: Xama. 
SILVA FRANKLIN, L. (2006). "Universidade: a ideia e a história”. Estudos Avançados, 20(56), 191-202.

SUÁREZ SOSAYA \& MUÑOZ GARCÍA. (2004). "Ruptura de la institucionalidad universitaria". En Ordorika, La academia en Jaque, perspectivas políticas sobre la evaluación de la educación superior en México (págs. 25-33). Distrito Federal, México: CRIM, UNAM.

UNAM. (2011). Portal de Estadística Universitaria. Recuperado el 28 de febrero de 2012, de http://www.estadistica.unam.mx/indicadores/

WEBOMETRICS. (2010). "Ranking Web of World Universities". Recuperado en octubre 14, 2010: http://www.webometrics.info/top100_continent.asp?cont=latin_america.

WILLIAMSON, J. (1990). “What Washington Means by Policy Reform”. En Latin American Adjustment: How Much Has Happened? Peterson Institute for International Economics.

EVANGELINA ZEPEDA GARCÍA: Doctora en Ciencias Políticas y Sociales por la Universidad Nacional Autónoma de México, en febrero de 2012. Coordinadora de la Maestría en Educación en la Universidad Marista de Querétaro.

E-mail: evazega@hotmail.com 